\title{
Allergy, Still Waiting to Explore: Recent Advancement in the Pathogenesis and Therapeutic Approaches of Allergy
}

\section{Peng-Yuan Zheng}

$5^{\text {th }}$ Affiliated Hospital of Zhengzhou University, Zhengzhou, Henan, China

*Corresponding author: Peng-Yuan Zheng, Professor of Medicine, 5th Affiliated Hospital of Zhengzhou University, Zhengzhou, Henan China, Tel: +86-371-65261035; Fax: +86-371-63934118; E-mail: medp7123@126.com

Received date: October 14, 2015; Accepted date: October 19, 2015; Published date: October 23, 2015

Copyright: (c) 2015 Zheng PY. This is an open-access article distributed under the terms of the Creative Commons Attribution License, which permits unrestricted use, distribution, and reproduction in any medium, provided the original author and source are credited.

\section{Editorial}

Allergic diseases increased greatly in recent decades and are becoming one of the major world health problems of this century. Why there is a high prevalence of allergic diseases not only in western world but in Asia and other developing countries still needs further investigation. It is a new frontier to explore the pathogenesis of allergy and its therapeutic approaches. Journal of Allergy \& Therapy is dedicated to allergic diseases and therapeutic researches from clinical trials and laboratory experiments, including various approaches adopted and new strategies in diagnosis, therapy and prevention of allergic diseases. I am pleased to write this editorial for Journal of Allergy \& Therapy and confident that this journal will become a highly respected resource in allergic diseases research.

The term "allergy" was first used in 1906, which derives from the Greek words "allos" and "ergos" to describe the hypersensitivity reactions of immune system to certain substances such as dust, pollen or some kind of foods. All forms of hypersensitivity used to be classified as allergies and the meaning of the word changed over time, allergy now is restricted to type I hypersensitivities (immediate hypersensitivity), which are mainly characterized as immunoglobulin E (IgE) antibodies mediated allergic diseases, including hay fever, food allergies, atopic dermatitis, allergic asthma and anaphylaxis, etc. Generally, two categories, namely host and environmental factors are responsible for this allergic condition. The host risk factors include heredity, sex, race and age that may put the individuals into a predisposition, namely atopy, which is an exaggerated tendency to become sensitized. The exposure to environmental pollution, infectious diseases and allergens makes up the external causes. Reduced exposure to environmental pathogenic microorganisms in the early childhood, according to the "hygiene hypothesis", results in insufficient stimulation of Th1 cells and leading to an overactive of Th2 cells polarization in immune system, which in turn facilitates allergic diseases. Other hypotheses from gut microbiota diversity, infant feeding, over exposure to allergens in occupation to the effects of pollution have been explored, while there is a complex interplay of environmental factors and host genetics that cannot be detached from each other in the immunopathogenesis of allergy, further efforts should be performed to elucidate the etiology of allergy.

With respect to the immunological mechanism involved in the allergic inflammation, allergy is generally categorized into three types: $\operatorname{IgE}$ mediated, mixed (IgE/non-IgE) and non-IgE mediated reactions. Allergy was extensively studied over years and the pathogenesis still keeps obscure. The IgE dependent type I hypersensitivity has two phases: Acute response, the body was sensitized in this stage, if later exposure to the same allergen, the acute response mediated by mast cells degranulation occurs, causing several allergic systemic effects, such as anaphylaxis, or localized to particular body systems, like intestine, respiratory tract and dermis. The late phase response may occur after the subsidence of acute response mediated by the migration of other leukocytes such as neutrophils and eosinophils.

IgE antibodies are key mediators of allergic diseases, which can bind to FceRI receptors on mast cells and basophils, responsible for symptoms of allergic diseases, so the production of $\operatorname{IgE}$ in B cells is tightly regulated by mechanisms of rapidly differentiate into plasma cells and highly apoptotic of IgE germinal center cells. The generation of IgE producing cells occurs after IgM and IgD expressing B cells were activated via class switch recombination, this B cells activation was regulated by cytokines from CD4 T cells such as IL-4/IL-13 and signals between CD40 on B cells and CD40L expressed on CD4 T cells interaction. $\mathrm{T}$ cell cytokine environment is very important for $\mathrm{IgE}$ production. IL-4/IL-13 signaling and STAT6 activation can promote IgE class switch recombination, while TGF- $\beta$ and IFN- $\gamma$ act directly on $\mathrm{B}$ cells to repress IgE production. Allergy is dominantly associated with Th2 cells skewed antigen-specific $\mathrm{T}$ cell profile. Th9 cells also can enhance mucin production and act as factors for mast cells recruitment and activation by secreting IL-9. In addition, dendritic cells (DC) play central role in programming $\mathrm{T}$ cells response and maintaining immune tolerance by altering their phenotypes after antigen processing and other signals stimulation. The default response to antigens is immune tolerance commonly through producing regulatory $\mathrm{T}$ cells. Pathogenic factors such as cholera toxin and staphylococcal enterotoxin may act as adjuvants to break this tolerance and lead to the generation of sensitization, while factors responsible for this corruption of immune tolerance and the mechanism of skewed Th2 cells proliferation in allergic inflammation still need further studied.

Allergic diseases impact the quality of life, reduce productivity and increase medical expenditures. In addition to avoidance of the provoking allergen, treatment of allergic diseases focuses on pharmacotherapy and immunotherapy. Allergen immunotherapies including subcutaneous immunotherapy (SCIT), sublingual immunotherapy (SLIT) and oral food allergen immunotherapy (OIT), which normally cause fewer symptoms by natural exposure can induce desensitization and modulate allergen specific allergic responses. Despite their widespread use, the underlying mechanisms keep still unclear and further efforts to evaluate the long-term effectiveness and safety for these new immunotherapies should be established. Studies about the underlying therapeutic mechanisms, clinical trials for desensitization rates, the eligibility and optimal dosing for immunotherapies should also be performed. Even some of the therapies are not ready for clinical use, researches continue into the use of many new immunotherapy strategies, which will be hopeful to revolutionize the existing treatment of allergic diseases. In addition, the development of recombinant technology in the field of allergy has 
Citation: Peng-Yuan Zheng (2015) Allergy, Still Waiting to Explore: Recent Advancement in the Pathogenesis and Therapeutic Approaches of Allergy. J Allergy Ther 6: e110. doi:10.4172/2155-6121.1000e110

Page 2 of 2

brought opportunities to the therapy of allergic diseases. Modified hypoallergenic allergens with reduced $\operatorname{IgE}$ binding capacity but retained $\mathrm{T}$ cell reactivity, $\mathrm{T}$ cell peptides that can stimulate allergen specific $\mathrm{T}$ cells and recombinant allergens have been investigated; Adjuvant therapies derived from bacterial origin to enhance the Th1 polarization such as CpG-oligodeoxynucleotides and new vaccines by fusing non-IgE reactive peptides from IgE binding sites to a virus protein to induce reduced $\operatorname{IgE}$ and $\mathrm{T}$ cell reactivity but can induce protective allergen specific IgG antibodies have delivered encouraging results.

Mast cells and basophils are effector cells in allergic response that release a large amount of mediators including serine proteinases, prostaglandin $\mathrm{D} 2$, proinflammatory cytokines etc. These mediators are increasingly becoming the targets for anti-allergic therapy other than traditional antihistamines and LTC4 blockers. Targeting their activating receptors such as high affinity IgE receptors and CD48 and the inhibitory receptors such as CD300a and FcyRIIb to develop new drugs, hold promising therapeutic possibilities. In addition, microbial exposures are critical regulators for the development of immune system, mainly via intestinal microbiota which can provides plenty of necessary antigenic stimuli. Association between intestinal microbiota and allergy has been well established and some selected probiotics are proved containing potential therapeutic effect to allergic diseases such as eczema, food allergy and asthma while the efficacy of probiotics requires further examination. 